\title{
Small and young radio sources
}

\author{
Monica Orienti ${ }^{* \dagger}$ \\ IAC - c/Via Lactea s/n, E-38205 La Laguna (Tenerife), Spain \\ E-mail: orienti@ira.inaf.it
}

It is currently accepted that compact and bright radio sources characterized by a convex spectrum peaking at frequencies ranging from $100 \mathrm{MHz}$ to a few $\mathrm{GHz}$ are young objects. In this scenario, high frequency peaker (HFP) radio sources, with a turnover frequency higher than $5 \mathrm{GHz}$ are good candidates to be extremely young radio sources with ages of up to a few thousand years. The knowledge of the conditions in young radio source is fundamental in order to draw reliable evolution models able to describe the entire life-cycle of the radio emission. Given the high spatial resolution and the large frequency range spanned, VLBI observations provide a unique opportunity to constrain the physical conditions in young radio sources, and to investigate the role played by the environment on the source growth. Recent VLBI results are discussed here.

The 9th European VLBI Network Symposium on The role of VLBI in the Golden Age for Radio Astronomy and EVN Users Meeting

September 23-26, 2008

Bologna, Italy

\footnotetext{
* Speaker.

${ }^{\dagger}$ This work has benefited from research funding from the European Community's sixth Framework Programme under RadioNet R113CT 20035058187
} 


\section{Small and young radio sources}

An active galactic nucleus (AGN) is a compact and luminous region hosted in the centre of a galaxy. Its luminosity, much higher than that observed in "non-active" galaxies, is not due to stellar emission, but it is related to accretion mechanisms on the central black hole. Only a small fraction of the AGN population $(\sim 10 \%)$ is radio-loud, making the origin of the radio emission a still open question.

The onset of radio emission is currently considered related to mergers or accretion events taking place in the host galaxy. In the early-stages of the radio source evolution, the radio emission has to make its way through the dense interstellar medium (ISM) before plunging into the smoother intracluster medium (ICM), and giving origin to extended (up to a few $\mathrm{Mpc}$ ) radio sources. In this scenario, the linear size of the radio source gives an indication of the evolution stage (i.e. the age) of the radio emission.

A significant fraction (15-30\%) of sources in flux-density limited radio catalogues is represented by powerful $\left(\mathrm{P}_{1.4 \mathrm{GHz}}>10^{25} \mathrm{~W} / \mathrm{Hz}\right)$ and intrinsically compact $\left(<1^{\prime \prime}\right)$ radio sources, with a convex synchrotron radio spectrum peaking at frequencies between $\sim 100 \mathrm{MHz}$ and a few GHz. If the peak of the spectrum occurs either at a few hundreds $\mathrm{MHz}$ or around one $\mathrm{GHz}$, the radio sources are identified as "compact steep spectrum (CSS) or "GHz-peaked spectrum" (GPS) respectively. When imaged with high spatial resolution, they usually display a two-sided morphology dominated by hot-spots and mini-lobes. For this reason, these radio sources are known as "Compact Symmetric Objects" (CSOs).

Their small size, together with their morphology, a scaled-down version $(0.1-15 \mathrm{kpc})$ of the extended classical radio galaxies, suggest that these objects represent a young stage in the individual radio source evolution (see e.g. [7,33]). This interpretation is supported by the detection of hot spot advance speed in a dozen of the most compact CSOs, leading to a kinematic age of about $10^{3}$ years [29]. The young age of CSOs has a further confirmation by means of spectral studies [18, 19] providing radiative ages of $10^{3}-10^{5}$ years.

The competing model, known as the frustration scenario, postulates that these objects are small because their are trapped by an extremely dense ambient medium able to frustrate the source growth [37]. However, no observational evidence of the presence of an unusually dense environment has been found $[8,32]$, giving an indirect support to the youth scenario.

The main property characterizing small and young radio sources is the presence of a peak in their synchrotron spectrum. This feature is commonly explained by means of synchrotron-self absorption (SSA) [33]. The empirical anti-correlation found between the linear size and the peak frequency [20] supports this view: as the source grows, its radio emission becomes optically-thin at lower and lower frequencies. This implies that the smallest, and thus youngest radio sources, must be sought among those with a spectrum peaking at high frequency, above a few GHz. With their spectral peak occurring well above 4-5 GHz, high frequency peakers (HFP) are the best candidate to be newly born radio sources [3, 4].

Since the selection of young radio sources is based mainly on the position of the spectral peak, it is possible that different kind of objects, like blazars may contaminate the samples. In this objects, the compact size is due to projection effects which foreshorten their real linear size, and it is not related to the source age. To discriminate between young and boosted objects, an accurate analysis 
of the radio properties is unavoidable.

\section{Radio properties}

\subsection{Spectral variability}

The presence of a convex spectrum with a peak occurring at different frequencies depending on the age of the source, is the main characteristic of young radio sources. In the presence of SSA, the peak is expected to move to lower frequency and the flux density in the optically-thick part of the spectrum should increase as the source grows in size, without indication of significant variability in the spectrum on small temporary scale. Multi-epoch observations showed that CSS, GPS and HFP sources identified with galaxies, are the least variable class of extragalactic objects $[21,34,23]$, while the majority of those identified with quasars show flux-density and spectralshape variability $[36,35]$. In a significant fraction of GPS/HFP quasars, the radio spectrum turns out to be flat in a subsequent observing epoch [36, 23], indicating a different nature for this class of objects.

\subsection{Morphology}

Given the sub-arcsecond size of young radio sources, the study of their structure became possible with the advent of VLBI. The first pioneering VLBI works on CSS/GPS sources revealed simple symmetric structures. Deeper observations allowed a more accurate description of their morphology [6]. Young radio sources, at least those identified with galaxies, have a "symmetric" structure dominated by two well-separated components with steep spectral index, interpreted as mini-lobes and/or hot spots, occasionally with a weak unresolved component in between, accounting for a small fraction $(<1 \%)$ of the flux density, usually detected at high frequency only, which may contain the source core (Fig. 1a). In this context, the term "symmetric" only refers to a two-sided morphology and does not imply that the source components have similar characteristics. Between the "symmetric" objects there are radio sources with highly asymmetric structures (Fig. 1b). In a significant fraction ( $65 \%$, [30]) of small radio galaxies, the two lobes have very different luminosity. Furthermore, when the source core is detected, it has been observed that also the separation ratio of the outer components from the nucleus is asymmetric, being the brightest also the closest to the core. Such asymmetries are more commonly found in small radio sources, than in large radio galaxies [1], suggesting an influence played by the ambient medium on the source growth.

On the other hand, small radio sources identified with quasars mainly display a one-sided Core-Jet morphology with a straight or bent structure always with a well visible core accounting for the majority of the flux density, and located at one edge of the source. The Core-Jet morphology is the structure usually displayed by beamed blazar objects. Small radio sources with such a morphology also show significant flux density and spectral-shape variability [23], suggesting, again, a different interpretation for the radio emission. 

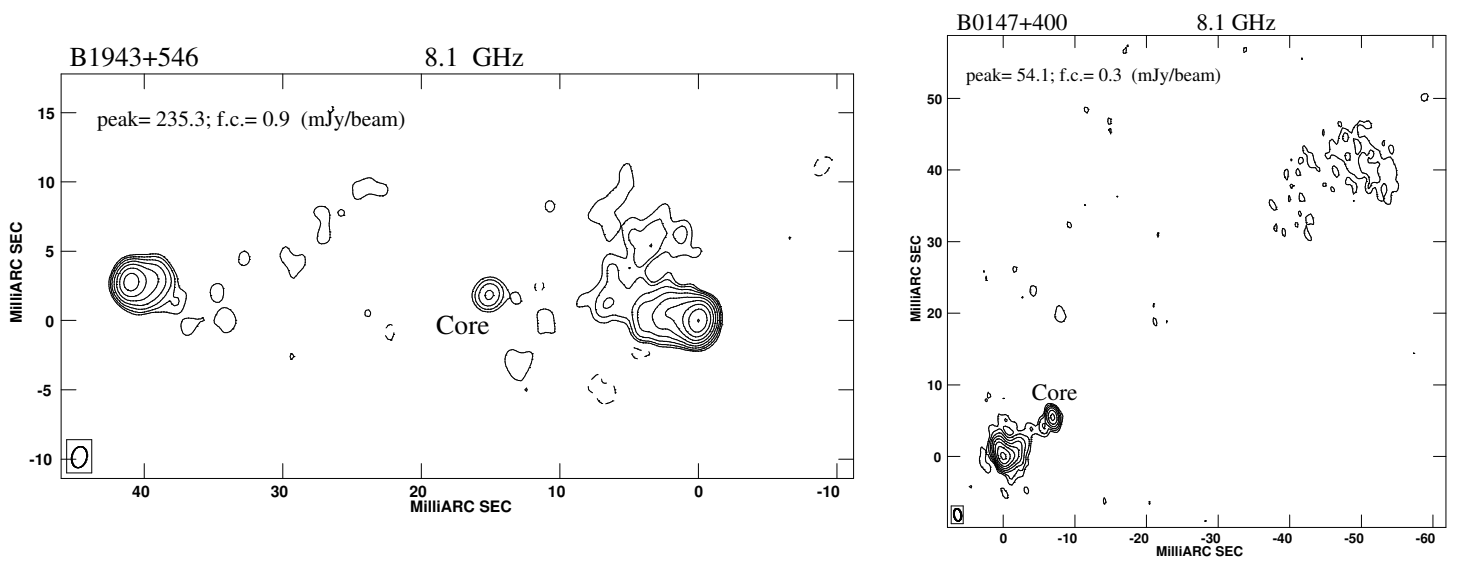

Figure 1: Example of "symmetric" structures in CSS/GPS radio sources.

\subsection{Polarization}

The study of the polarization enables us to derive the distribution of the magnetic field in young radio sources. However, young radio sources completely reside within the ISM of the host galaxy. This gas can act as a Faraday Screen causing substantial depolarization of the synchrotron radiation.

In large ( $>5 \mathrm{kpc})$ CSS objects, the radiation has high percentage of polarization $(>10 \%,[10])$ and the magnetic field is generally parallel to the jet axis [9], as found in edge-brightened large radio galaxies. On the other hand, radio galaxies smaller than $5 \mathrm{kpc}$ are unpolarized or possess very low percentage of polarized emission [10, 24]. The high rotation measures (RM) found suggests that the radiation is strongly depolarized by the magneto-ionic plasma of the ambient medium.

As found for variability and morphological properties, a different behaviour is shown by GPS and HFP quasars, where high values of polarized emission are detected [24, 34].

The different characteristics shown by small radio sources with different optical identification suggest that small radio objects hosted either by galaxies or by quasars represent two different radio source populations: genuinely young radio objects the former, beamed blazars the latter.

\section{Physical conditions}

The radio emission of extragalactic objects is due to synchrotron radiation produced by relativistic electrons with a power-law energy distribution. A curvature in the spectrum produced by synchrotron self-absorption mechanism is thus present by default, and the frequency at which it occurs is strongly related to the size of the radio component. In the context of evolution models, this explain the anti-correlation found between peak frequency and linear size: as the source expands, the synchrotron radiation becomes optically thin at lower energy, shifting the radio spectrum towards lower frequency.

In order to draw a complete picture able to describe the various stages of the radio source evolution, a deep knowledge of the physical conditions in young radio sources is necessary. Evolution models 
proposed so far, e.g. [7, 33], are based on the assumption of minimum energy conditions, corresponding to a near equipartition of energy between particles and magnetic field [26]. Equipartition magnetic fields derived in young radio sources ranges from a few $\mathrm{mG}$, in the compact components of CSS [7], up to tens or hundreds $\mathrm{mG}$ in HFPs [25]. However, there is no a-priori reason for believing that magnetic fields are those of equipartition, and it is important to derive the magnetic field in an independent way.

If the spectral peak is due to SSA as stated before, the magnetic field $B$ of a homogeneous synchrotron component can be computed by observable quantities only:

$$
B=f(\alpha)^{-5} \theta^{4} v_{\max }^{5} S_{\max }^{-2}(1+z)^{-1}
$$

where $\theta$ is the source angular size, $S_{\max }$ is the peak flux density, $v_{\max }$ the peak frequency, $z$ is the redshift and $f(\alpha)$ is a function of the spectral index [12].

The main difficulty in applying this method is the uncertainty in determining the source parameters, in particular the peak frequency and the angular size. For an accurate determination of the peak frequency, the radio spectrum must be well sampled in both its optically-thick and -thin parts. Furthermore, the magnetic field should not be computed on the whole source, but on individual sub-structures, which are a better approximation of homogeneous components.

The VLBI has the ideal frequency coverage and spatial resolution to perform such an investigation. Magnetic fields derived from the spectral peak of CSS radio sources were within a factor of 16 of the equipartition values [31]. The discrepancy between the values may arise from the uncertainties in the determination of the spectral peak which occurs at rather low frequencies where the sampling is more difficult and size determination more critical.

In HFP objects, the spectral peak at a few GHz allows an accurate determination of the peak parameters. Furthermore, given their compact size due to the severe radiative losses which prevent the formation of extended lobe-like features, HFP sub-structures can be considered a good approximation of uniform synchrotron components. The magnetic fields inferred are in good agreement with those computed assuming equipartition, with a few exception [25]. In some HFP components, the magnetic fields derived are above a few Gauss, that is one or two orders of magnitude higher than the equipartition value. However, in these components it was noted that the optically-thick part of the spectrum is extremely inverted with a spectral index $\alpha<-2.5\left(S \propto v^{-\alpha}\right)$, i.e. the canonical value of SSA, implying the presence of an additional contribution from free-free absorption (FFA). In this case, the magnetic fields derived by means of the peak parameters are physically meaningless.

\section{Ambient medium}

The origin of radio activity in AGN is thought to be related to the availability of fuel to feed the central engine, likely provided by merger events. For this reason, galaxies hosting a radio source in an early evolutionary stage, are supposed to possess a rich and dense interstellar medium. The presence of significant amount of gas in young radio sources was proved by the high incidence of HI absorption [28], which is substantially higher than what typically found in large (hundreds of 
$\mathrm{kpc}$ in size) radio galaxies [15].

Statistical studies of the properties of the HI absorption in young CSS/GPS objects pinpointed the existence of an anti-correlation between the HI column density and the linear size [28, 11]: the smaller the source, the larger the HI column density. This result can be explained assuming that the neutral hydrogen is organized in a circumnuclear structure, such as a torus or a disk. In this context, the HI absorption is detected against the receading jet, when our line of sight passes through the circumnuclear disk/torus along its way towards the radio emission [17]. As the source grows, the background emission of the counter-jet moves toward the outer and less dense part of the atomic structure, causing a decrement of the HI column density.

Surprisingly, the anti-correlation found in CSS/GPS objects is not present in the small HFP sources [22]. This unexpected result can be explained again in a torus scenario. Given the extremely small size of HFPs, the line of sight to the counter-jet passes mainly through the ionized part of the ISM, piercing the atomic structure in its innermost part, where it is hotter and less dense. Support to this interpretation arises from the detection of large amount gas in HFPs [5, 25], responsible for free-free absorption.

The anti-correlation between linear size and HI column density was derived by observations with a spatial resolution not adequate to determine the distribution of the atomic gas. To test whether the neutral hydrogen is settled in a circumnuclear structure, the high spatial resolution provided by VLBI observations are needed.

In the GPS sources 3C 31.04 [2] and 1946+708 [27] a torus-like structure has been detected. On the other hand, in the GPS sources 4C 12.50 [16] and 0402+379 [14], and in the two very asymmetric CSS 3C 49 and 3C 268.3 [13], VLBI observations showed that HI absorption occurs far away from the centre, indicating that the absorption is likely due to an off-nuclear cloud. Furthermore, in 3C 49 and 3C 268.3, HI absorption is detected only against the brightest lobe, which turned out to be also the closest to the core. This suggests that jet-cloud interactions may take place, at least when the radio emission is still growing within the ISM of the host galaxies. Such an impact may slow down the propagation of the interacting jet and enhance its luminosity, explaining in this way the strong luminosity an arm-length asymmetries observed in many young radio sources.

\section{Conclusions}

Flux density limited catalogues are known to possess a significant fraction of small radio sources showing a peak in their synchrotron radio spectra. Their small size, together with their two-sided morphology, a scaled-down version of the structures shown by large radio galaxies, suggest that these radio sources represent an early stage in the evolution of the radio emission. The study of their radio properties, namely the spectral variability, morphology and polarization, pointed out that those objects optically identified with galaxies are genuinely young radio sources, while the majority of those identified with quasars are likely boosted blazar objects.

The presence of a spectral peak is the main characteristic of young radio sources. This peak is due to SSA, although an additional contribution from FFA has been found in the most compact objects. Where the spectral peak is originated by SSA only, it is possible to derive the magnetic field by means of the peak parameters. Magnetic fields computed in this way are generally in good agreement with those calculated assuming equipartition of energy between particles and magnetic 
field, providing a strong evidence that young radio sources are in minimum energy conditions. The presence of a dense and inhomogeneous ambient medium surrounding young radio sources has been pinpointed by frequent detection of neutral hydrogen in absorption. Although in many cases the atomic gas is organized in settled, circumnuclear structures as disks and tori, VLBI observations showed the presence of unsettled, off-nuclear atomic clouds, responsible for HI absorption in the outer regions of the radio sources. The interaction between one of the jet and an off-nuclear cloud may influence the source growth, for example slowing down the source expansion, and producing asymmetric structures.

Future telescopes with good sensitivity and resolution like SKA, will provide us with new information on the source environment, that is a fundamental ingredient for the definition of a complete picture of the radio source evolution.

\section{References}

[1] Arshakian, T.G., Longair, M.S., 2000, MNRAS, 311, 846

[2] Conway, J.E., 1996, IAUS, 175, 92

[3] Dallacasa, D., Stanghellini, C., Centonza, M., Fanti, R., 2000, A\&A, 363, 887

[4] Dallacasa, D., 2003, PASA, 20, 79

[5] Dallacasa, D., Orienti, M., 2009, AN, in press

[6] Fanti, R., Fanti, C., Schilizzi, R.T., et al., 1990, A\&A, 231, 333

[7] Fanti, C., Fanti, R., Dallacasa, D., Schilizzi, R.T., Spencer, R.E., Stanghellini, C., 1995, A\&A, 302, 31

[8] Fanti, C., Pozzi, F., Fanti, R., et al., 2000, A\&A, 358, 449

[9] Fanti, C., Pozzi, F., Dallacasa, D., Fanti, R., Gregorini, L., Stanghellini, C., Vigotti, M., 2001, A\&A, 369,380

[10] Fanti, C., Branchesi, M., Cotton, W.D., et al., 2004, A\&A, 427, 465

[11] Gupta, N., Salter, C.J., Saikia, D.J., Ghosh, T., Jeyakumar, S., 2006, MNRAS, 373, 972

[12] Kellermann, K.I., Pauliny-Toth, I.I.K., 1981, ARA\&A, 19, 373

[13] Labiano, A., Vermeulen, R., Barthet, P.D., et al., 2006, A\&A, 447, 481

[14] Maness, H.L., Taylor, G.B., Zavala, R.T., Peck, A.B., Pollack, L.K., 2004, ApJ, 602, 123

[15] Morganti, M., Oosterloo, T.A., Tadhunter, C.N., van Moorsel, G., Killeen, N., Wills, K.A., 2001, MNRAS, 323, 331

[16] Morganti, R., Oosterloo, T.A., Tadhunter, C.N., Vermeulen, R., Pihlström, Y.M., van Moorsel, G., Wills, K.A., 2004, A\&A, 424, 119

[17] Mundell, C.G., Wrobel, J.M., Pedlar, A., Gallimore, J.F., 2003, ApJ, 583, 192

[18] Murgia, M., Fanti, C., Fanti, R., Gregorini, L., Klein, U., Mack, K.-H., Vigotti, M., 1999, A\&A, 345, 769

[19] Murgia, M., 2003, PASA, 20, 19 
[20] O’Dea, C.P, Baum, S.A., 1997, AJ, 113, 148

[21] O’Dea, C.P., 1998, PASP, 110, 493

[22] Orienti, M., Morganti, R., Dallacasa, D., A\&A, 457, 531

[23] Orienti, M., Dallacasa, D., Stanghellini, C., 2007, A\&A, 475, 813

[24] Orienti, M., Dallacasa, D., 2008, A\&A, 479, 409

[25] Orienti, M., Dallacasa, D., 2008, A\&A, 487, 885

[26] Pacholczyk, A.G., 1970, Radio Astrophysics (San Francisco: Freeman \& Co.)

[27] Peck, A.B., Taylor, G.B., Conway, J.E., 1999, ApJ, 521, 103

[28] Pihlström, Y.M., Conway, J.E., Vermeulen, R.C., 2003, A\&A, 404, 871

[29] Polatidis, A.CG, Conway, J.E., 2003, PASA, 20, 69

[30] Saikia, D.J., jeyakumar, S., Mantovani, F., Salter, C.J., Spencer, R.E., Thomasson, P., Wiita, J., 2003, PASA, 20, 50

[31] Scott, M.A., Readhead, A.C.S., 1977, MNRAS, 180, 539

[32] Siemiginowska, A., Cheung, C.C., LaMassa, S., Burke, D.J., Aldcroft, T.L., Bechtold, J., Elvis, M., Worrall, D.M., 2005, ApJ, 632, 110

[33] Snellen, I.A.G., Schilizzi, R.T., Miley, G.K., de Bruyn, A.G., Bremer, M.N., Röttgering, H.J.A., 2000, MNRAS, 319, 445

[34] Stanghellini, C., O’Dea, C.P., Dallacasa, D., Baum, S.A., Fanti, R., Fanti, C., 1998, A\&AS, 131, 303

[35] Tinti, S., Dallacasa, D., de Zotti, G., Celotti, A., Stanghellini, C., 2005, A\&A, 432, 31

[36] Torniainen, I., Tornikoski, M., Teräsranta, H., Aller, M.F., Aller, H.D., 2005, A\&A, 435, 839

[37] van Breugel, W., Miley, G., heckman, T., 1984, AJ, 89, 5 